\title{
Addendum
}

\section{Reactions of $\boldsymbol{p}$-Benzoquinone with Sulfur Nucleophiles}

Alan R. Katritzky,* Dmytro Fedoseyenko, Prabhu P. Mohapatra, Peter J. Steel Synthesis 2008, 777.

Compound 14a is a known compound but was not reported as such. Göksel et al.'s 2005 paper should have been cited in connection with compound 14a:

Göksel, F. S.; İbiş, C.; Bayrak, N. A. Phosphorus, Sulfur Silicon Relat. Elem. 2005, 180, 1961.

The authors apologize for the initial omission of the reference. 